PREPARED FOR THE U.S. DEPARTMENT OF ENERGY, UNDER CONTRACT DE-AC02-76CH03073

PPPL-3555

PPPL-3555

UC-70

Gyrokinetic Calculations of the Neoclassical Radial Electric Field in Stellarator Plasmas

by

J.L.V. Lewandowski, J. Williams, A.H. Boozer, and Z. Lin

April 2001

NM|

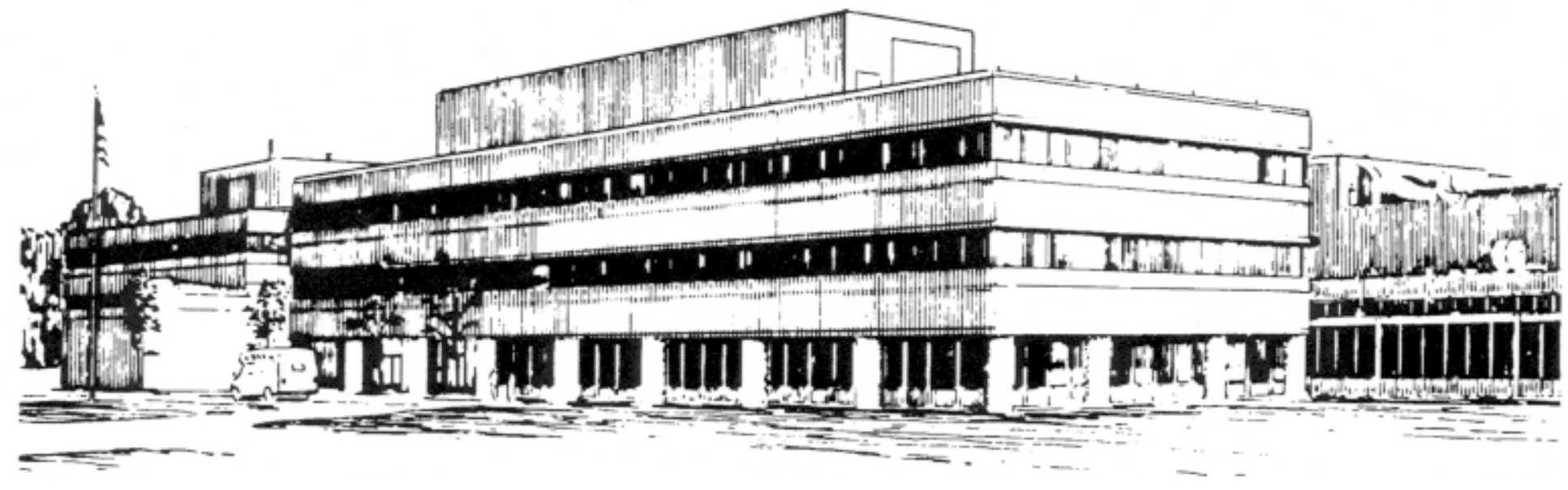

PRINCETON PLASMA PHYSICS LABORATORY PRINCETON UNIVERSITY, PRINCETON, NEW JERSEY 


\section{PPPL Reports Disclaimer}

This report was prepared as an account of work sponsored by an agency of the United States Government. Neither the United States Government nor any agency thereof, nor any of their employees, makes any warranty, express or implied, or assumes any legal liability or responsibility for the accuracy, completeness, or usefulness of any information, apparatus, product, or process disclosed, or represents that its use would not infringe privately owned rights. Reference herein to any specific commercial product, process, or service by trade name, trademark, manufacturer, or otherwise, does not necessarily constitute or imply its endorsement, recommendation, or favoring by the United States Government or any agency thereof. The views and opinions of authors expressed herein do not necessarily state or reflect those of the United States Government or any agency thereof.

\section{Availability}

This report is posted on the U.S. Department of Energy's Princeton Plasma Physics Laboratory Publications and Reports web site in Calendar Year 2001. The home page for PPPL Reports and Publications is: http://www.pppl.gov/pub_report/

DOE and DOE Contractors can obtain copies of this report from:

U.S. Department of Energy

Office of Scientific and Technical Information

DOE Technical Information Services (DTIS)

P.O. Box 62

Oak Ridge, TN 37831

Telephone: (865) 576-8401

Fax: (865) 576-5728

Email: reports@adonis.osti.gov

This report is available to the general public from:

National Technical Information Service

U.S. Department of Commerce

5285 Port Royal Road

Springfield, VA 22161

Telephone: 1-800-553-6847 or

(703) 605-6000

Fax: (703) 321-8547

Internet: http://www.ntis.gov/ordering.htm 


\title{
Gyrokinetic Calculations of the Neoclassical Radial Electric Field in Stellarator Plasmas
}

\author{
J.L.V. Lewandowski ${ }^{(1)}$, J. Williams ${ }^{(2)}$, A.H. Boozer ${ }^{(2)}$ and Z. Lin ${ }^{(1)}$
}

(1) Plasma Physics Laboratory, Princeton University, P.O. Box 451, Princeton NJ 08543

(2) Department of Applied Physics, Columbia University, New York, NY 10027

(February 26, 2001)

\begin{abstract}
A novel method to calculate the neoclassical radial electric field in stellarator plasmas is described. The method, which does not have the inconvenient of large statistical fluctuations (noise) of standard Monte Carlo technique, is based on the variation of the combined parallel and perpendicular pressures on a magnetic surface. Using a three-dimensional gyro-kinetic $\delta f$ code, the calculation of the radial electric field $\left(E_{r}\right)$ in the National Compact Stellarator Experiment has been carried out. It is shown that a direct evaluation of $E_{r}$ based on a direct calculation of the radial particle flux is not tractable due to the considerable noise.
\end{abstract}

Pacs \# : 52.35Kt, 52.30Jb, 52.35Ra 


\section{INTRODUCTION}

The lack of toroidal symmetry of stellarators requires a fully 3-dimensional description of the plasma. The departure from axi-symmetry in stellarator plasmas leads to enhanced neoclassical losses in the low-collisionality regime. Another related feature of the effect of the non-axisymmetry of the plasma is to strongly modify the drift orbits of the particles (see the reviews by Sadgdeev and Galeev ${ }^{1}$, and by Kovrizhnykh ${ }^{2}$ ).

Over the past few years, there has been a renewed interest in the so-called quasi-axisymmetric (QA) stellarator concept; that is the equilibrium magnetic field strength is approximately symmetric in the magnetic toroidal angle $\zeta$, after transformation to Boozer coordinates ${ }^{3}$. A stellarator experiment based on the QA concepts is currently being designed in the United States $^{4}$; the National Compact Stellarator Experiment $(\mathrm{NCSX})^{5}$ is a three-field period, low-aspect ratio configuration which has good transport and stability properties ${ }^{5-7}$. One important feature of the QA concept is that the plasma can rotate in the direction of quasi-axisymmetry, and it may be possible to exploit and control the formation of transport barriers, as in advanced tokamak plasmas ${ }^{8}$. Since the radial electric field is a major contender ${ }^{9}$ in the formation of transport barriers, an accurate calculation of the radial electric field is an important aspect of QA plasmas. The reader who is not familiar with current trends in stellarator design can consult Ref.[10].

In this paper we describe a novel method to calculate the neoclassical radial electric field in asymmetric toroidal plasmas. The low-noise method, which exploits the advantages of the $\delta f$ algorithm ${ }^{11-14}$, is based on the variation of the combined parallel and perpendicular

pressures $\left(P_{\|}\right.$and $\left.P_{\perp}\right)$ on a magnetic surface. As an example, we have calculated the radial electric field $E_{r}$ for the C82 configuration of the NCSX plasma ${ }^{5}$. It is shown that a direct (Monte Carlo) calculation of the radial electric field based on the radial particle flux is not tractable due to the large statistical fluctuations; interestingly, the gyrokinetic calculation 
using the variation of $P_{\|}$and $P_{\perp}$ on a given magnetic surface does not exhibit large statistical deviations, which allows for a determination of $E_{r}$.

The paper is organized as follows; in section 2, we describe the method used to calculate the radial particle flux. The numerical method, the computational details and the results are given in section 3. Concluding remarks and future application of the method are presented in section 4.

\section{THE METHOD}

In this section, we describe the method used to determine the radial electric field based on the local variation of the parallel and perpendicular pressures ( $P_{\|}$and $P_{\perp}$, respectively). The quantities $P_{\|}$and $P_{\perp}$ are evaluated by taking appropriate velocity moments of $\delta f \equiv f-f_{0}$, where $f$ is the total distribution function whereas $f_{0}$ is its equilibrium part (usually $f_{0}$ is taken to be a Maxwellian distribution). The perturbed part of the distribution function, $\delta f$, evolves due to the combined effects of magnetic drifts and spatial inhomogeneity. Another subtle point regarding the numerical calculation is that the velocity moments for $P_{\|}$and $P_{\perp}$ are carried out in small annulus (i.e. finite volume) around a magnetic surface of reference; this point is discussed in more detail in section 3.

In stellarator geometry, it is convenient, both for analytical and computational purposes, to use magnetic coordinates. The confining magnetic field $\mathbf{B}$ is written in Boozer coordinates ${ }^{3}$ as

$$
\begin{aligned}
& \mathbf{B}=\iota(\psi) \nabla \zeta \times \nabla \psi+\nabla \psi \times \nabla \theta \\
& \mathbf{B}=g(\psi) \nabla \zeta+I(\psi) \nabla \theta+\beta_{\star} \nabla \psi
\end{aligned}
$$

where $\theta$ and $\zeta$ are the poloidal and toroidal angles, respectively; $\psi$ is proportional to the en-

closed toroidal flux; $\iota$ is the rotational transform; $g(\psi)$ and $I(\psi)$ are, within a multiplicative constant, the poloidal and toroidal currents, respectively. In these coordinates the Jacobian 
of the transformation, $\mathcal{J} \equiv[\nabla \psi \cdot(\nabla \theta \times \nabla \zeta)]^{-1}$, satisfies $\mathcal{J} B^{2}=g(\psi)+\iota(\psi) I(\psi) \equiv F(\psi)$. Consider the momentum of the balance equation for the ions

$$
\rho \frac{d \mathbf{V}}{d t}=-\boldsymbol{\nabla} \cdot \mathbf{P}+e n\left(\mathbf{E}+\frac{\mathbf{V} \times \mathbf{B}}{c}\right)+\mathbf{F}+\mathbf{R}
$$

where $\mathbf{V}$ is the fluid velocity, $\rho$ is the mass density, $\mathbf{R}$ is the force due to collisions, $\mathbf{F}$ represents the (possible) applied force, and $\mathbf{P}=P_{\|} \widehat{\mathbf{b}} \widehat{\mathbf{b}}+(\mathbf{I}-\widehat{\mathbf{b}} \widehat{\mathbf{b}}) P_{\perp}$ is the pressure tensor; here $\widehat{\mathbf{b}}=\mathbf{B} / B$ is the unit vector along $\mathbf{B}, P_{\|}$and $P_{\perp}$ are, respectively, the parallel and perpendicular pressures. We note that Eq. (2) can be obtained by taking the first-order velocity moment of the kinetic equation in the small gyro-radius limit. Taking the scalar product of $\mathbf{e}_{\zeta} \equiv \partial \mathbf{r} / \partial \zeta$ (where $\mathbf{r}$ is the position vector) with Eq.(2), and operating with $\langle\ldots\rangle=\iint \ldots \mathcal{J}(\psi, \theta, \zeta) d \theta d \zeta$, we obtain

$$
\frac{\iota(\psi)}{c} \frac{d Q}{d t}-\left\langle\frac{d L_{\zeta}}{d t}\right\rangle=\left\langle\frac{\partial \widehat{P}}{\partial \zeta}\right\rangle-T_{\zeta}
$$

where $\widehat{P} \equiv\left(P_{\|}+P_{\perp}\right) / 2, T_{\zeta}=\left\langle(\mathbf{R}+\mathbf{F}) \cdot \mathbf{e}_{\zeta}\right\rangle$ is the torque due to applied forces and collisional drag; $L_{\zeta}$ is the toroidal component of the canonical momentum $\mathbf{L}=\rho \mathbf{V}+e \mathbf{A} / c$ where $\mathbf{A}=\psi \boldsymbol{\nabla} \theta-\chi \boldsymbol{\nabla} \zeta$ is the vector potential and $2 \pi \chi$ is the poloidal flux. In deriving Eq.(3) we have assumed that the electrostatic potential (yet to be determined) is of the form $\Phi=\Phi(\psi)$, and we have neglected the loop voltage $(\partial \chi / \partial t \simeq 0)$. Using Eq.(1), the first term on the left-hand side of Eq.(3) can be obtained from

$$
\left\langle e n \mathbf{e}_{\zeta} \cdot\left(\frac{\mathbf{V} \times \mathbf{B}}{c}\right)\right\rangle=\frac{e}{c} \iint n \mathbf{V} \cdot\left(\mathbf{B} \times \mathbf{e}_{\zeta}\right) \mathcal{J} d \theta d \zeta=\frac{e}{c} \iota(\psi) \int \boldsymbol{\Gamma} \cdot d \boldsymbol{\sigma}_{n}=\frac{\iota(\psi)}{c} \frac{d Q}{d t}
$$

Here $Q$ is the total charge, $\boldsymbol{\Gamma}=n \mathbf{V}$ is the particle flux, and $d \boldsymbol{\sigma}_{n} \equiv \mathcal{J} \boldsymbol{\nabla} \psi d \theta d \zeta$ is an area element normal to the magnetic surface $\psi=$ const and pointing outwards. The first term on the right-hand side of Eq.(3), which is related to the pressure tensor term in Eq.(2), is derived in Appendix A. The parallel and perpendicular pressures which enter

$$
S \equiv\langle\partial \widehat{P} / \partial \zeta\rangle
$$

are calculated from the velocity moments of $\delta f$ which, in turn, depends on the particle trajectories. The particle trajectories being affected by the applied electric field 
$\mathbf{E}=-(d \Phi / d \psi) \nabla \psi$, we may write $S=S\left(E_{r}, t\right)$. After a few collision times the distribution $\delta f$ will relax and $S_{\infty}\left(E_{r}\right)=S\left(E_{r}, t \mapsto \infty\right)$ will provide a measure of the time variation of the charge $Q$. For one-species simulation, one can then determine the radial electric $E_{r}^{(0)}$ by solving

$$
S_{\infty}\left[E_{r}^{(0)}\right]=0
$$

Alternatively, one can measure the flux surface-averaged radial particle flux $\left\langle\Gamma_{r}\right\rangle\left(E_{r}\right)$ and determine $E_{r}^{(0)}$ such that the particle flux vanishes. However in quasi-axisymmetric toroidal configuration, the large statistical fluctuations in $\Gamma_{r}$ can be comparable to, or larger than, the time-averaged signal. As will be shown in the next section, a direct measurement of the radial particle flux is too noisy to be of practical use. Alternatively a dynamic calculation using the global gyrokinetic toroidal code $(\mathrm{GTC})^{15}$, which has been rigorously benchmarked against analytical tokamak neoclassical transport theory ${ }^{16}$, shows that $S\left(E_{r}, t\right)$ reaches a low-noise asymptotic value after a few ion-ion collision times. The general behavior of $S$ is of the form $S\left(E_{r}, t\right) \simeq S_{0} \exp \left(-C t / \tau_{i i}\right)+S_{\infty}\left[1-\exp \left(-C t / \tau_{i i}\right)\right]$, where $\tau_{i i}$ is the ionion collision time, $S_{0}\left(E_{r}\right)=S\left(E_{r}, t=0\right)$, and $C$ is a constant of the order of unity. It is interesting to note that $S$ provides information on the asymmetric part of the particle transport. As it turns out, $S$ strictly vanish in an axi-symmetric configuration (such as an ideal, 2-dimensional tokamak plasma). To show this, we use the definition of the flux-surface average and perform an integration by parts

$$
S=-\iint \widehat{P} \frac{\partial \mathcal{J}}{\partial \zeta} d \theta d \zeta=-\left\langle\widehat{P} \mathcal{J}^{-1} \frac{\partial \mathcal{J}}{\partial \zeta}\right\rangle
$$

Noting that $\mathcal{J} B^{2}$ is a flux surface quantity it follows that $\mathcal{J}^{-1} \partial \mathcal{J} / \partial \zeta=-2 B^{-1} \partial B / \partial \zeta$ and

$$
S=\left\langle\frac{P_{\|}+P_{\perp}}{B} \frac{\partial B}{\partial \zeta}\right\rangle
$$

showing that $S \equiv 0$ in an axi-symmetric configuration. We note that the off-diagonal contributions in the pressure tensor have been neglected (in terms of the smallness parameter $\left.\rho_{i} / R_{0}\right)$. The inclusion of finite Larmor radius (FLR) effects, such as in the paper of Rosenbluth et al. ${ }^{18}$, can lead to non ambipolar transport. 


\section{NUMERICAL METHOD AND RESULTS}

In this section, we describe the low-noise numerical method used to evaluate $S\left(E_{r}, t\right)$, which provides a measure of the radial particle flux. The computational domain and the collision operators are also discussed. Finally we present specific numerical results for the NCSX plasma.

It is convenient to write the parallel and perpendicular pressures in terms of their respective Fourier components (on a given magnetic surface); for example, the perpendicular pressure can be written as

$$
P_{\perp}=\sum_{m, n}\left(P_{\perp}\right)_{m, n} \exp \left[i\left(m \theta+n N_{p} \zeta\right)\right]
$$

where $N_{p}$ is the number of field periods of the configuration and the Fourier coefficients are calculated according to

$$
\left(P_{\perp}\right)_{m, n}=\frac{\int_{0}^{2 \pi} d \theta \int_{0}^{2 \pi} d \zeta\left(m v_{\perp}^{2} / 2\right) \delta f \exp \left[-i\left(m \theta+n N_{p} \zeta\right)\right] d^{3} v}{\int_{0}^{2 \pi} d \theta \int_{0}^{2 \pi} d \zeta}
$$

In practise the particle trajectories are integrated in a volume enclosed between two neighboring toroidal flux surfaces $\bar{\psi}-\Delta \psi / 2$ and $\bar{\psi}+\Delta \psi / 2$. (Fig.1). Here $\bar{\psi}$ is a magnetic surface of reference and $\Delta \psi$ is chosen so that $\Delta \psi / \psi_{b}$, where $\psi_{b}$ is the toroidal flux at the plasma boundary, is much less than unity. However, due to the combined effects of magnetic drifts and background inhomogeneity, the particles will drift outside the layer $\Delta \psi$, the number of Lagrangian markers within the layer will decrease in time, and the statistics associated with $P_{\|}$and $P_{\perp}$ will become poorer. To bypass this difficulty, particles are uniformly loaded in the layer $\Delta \psi$ but the perpendicular and parallel pressures are monitored in the annulus $\delta \psi \ll \Delta \psi$ (Fig.2). Introducing the radial coordinate $r=\sqrt{\psi / B_{0}}$ (where as before $\psi$ is the enclosed toroidal flux), one has $\Delta \psi=\psi(r+\Delta r)-\psi(r) \approx 2 B_{0} r \Delta r$ from which we get $\Delta r \approx \Delta \psi /\left(2 \sqrt{B_{0} \psi}\right)$. The radial component of the curvature drift velocity is of the order of $V_{d} \approx v_{t h}\left(\rho_{t h} / R\right)$, where $v_{t h}$ is the thermal velocity, $\rho_{t h}$ is the associated thermal gyroradius and $R$ is the major radius; here we have used $R_{c} \equiv|\nabla B / B|^{-1} \sim R$ as the typical scale length 
of the magnetic field inhomogeneity. Therefore the typical drift time within the layer $\Delta \psi$ is $\tau_{d} \approx \Delta r / V_{d}$, which is chosen so that $\tau_{d} \gg \tau_{r}$, where $\tau_{r}$ is the relaxation time (typically a few ion-ion collision time). Alternatively, one can assume periodicity in the radial direction, that is the particle that escapes the radial domain $\delta \psi$ can be put back in the same domain; in this case, the number of Lagrangian markers remains constant. Different schemes can be used and compared to randomize the position $(\psi, \theta, \zeta)$ and the pitch of the particle. However, as in a real experiment, one is interested in the global radial profile of the electric field; one important aspect of the method described above is that it can easily be extended to the entire plasma volume, without additional assumptions regarding the boundary conditions.

The guiding center motion and the collisions will spread the particles toward equal density in pitch and over the magnetic surface; therefore it is convenient to make the replacement (again using the particular form of the Jacobian in Boozer coordinates and the definition of the volume element in magnetic coordinates, $\left.d^{3} x=\mathcal{J} d \psi d \theta d \zeta\right)$

$$
\iint d \theta d \zeta \Longrightarrow \int \mathcal{J}^{-1}(\delta \psi)^{-1} d^{3} x \Longrightarrow[F(\bar{\psi}) \delta \psi]^{-1} \int B^{2} d^{3} x
$$

Therefore one can calculate the Fourier coefficients for the perpendicular pressure according to

$$
\left(P_{\perp}\right)_{m, n}=\int \frac{\int d^{3} x\left(m v_{\perp}^{2} / 2\right) \delta f B^{2} \exp \left[-i\left(m \theta+n N_{p} \zeta\right)\right]}{\int d^{3} x B^{2}} d^{3} v
$$

The same method to evaluated to the parallel pressure on the magnetic surface $\bar{\psi}$.

As is well known in neoclassical theory, the momentum and energy conservation properties of the collision operator are important for accurate calculation of quantities such as the radial particle flux. The gyrophased collision operator for like-species collisions can be written as $^{17}$

$C(\delta f)=\frac{\partial}{\partial v_{\|}}\left(\nu_{\|} \delta f\right)+\frac{\partial}{\partial v_{\perp}^{2}}\left(\nu_{\perp} \delta f\right)+\frac{\partial^{2}}{\partial v_{\|} v_{\perp}^{2}}\left(\nu_{\perp}^{\|} \delta f\right)+\frac{\partial^{2}}{\partial v_{\|}^{2}}\left(\nu_{\|}^{\|} \delta f\right)+\frac{\partial^{2}}{\left(\partial v_{\perp}^{2}\right)^{2}}\left(\nu_{\perp}^{\perp} \delta f\right)$ 
where

$$
\begin{aligned}
\nu_{\|} & =\nu_{0} v_{\|} F \\
\nu_{\perp} & =\nu_{0}\left[v_{\perp}^{2}(2 F-H-G)-2 v_{\|}^{2} G\right] \\
\nu_{\perp}^{\|} & =2 \nu_{0} v_{\perp}^{2} v_{\|}(H-G) \\
\nu_{\|}^{\|} & =\nu_{0}\left(v_{\|}^{2} H / 2+v_{\perp}^{2} G / 2\right) \\
\nu_{\perp}^{\perp} & =2 \nu_{0} v_{\perp}^{2}\left(v_{\perp}^{2} H+v_{\|}^{2} G\right)
\end{aligned}
$$

In Eq.(10), $\nu_{0}=4 \pi n_{\beta} q_{\alpha}^{2} q_{\beta}^{2} \ln \Lambda_{\alpha \beta} /\left(m_{\alpha}^{2} v^{3}\right)$ is the basic frequency for collisions of test particles $\alpha$ with background particle $\beta$. F, G and $H$ are dimensionless functions that can be written in terms of the Maxwell integral $\Theta(x)=2 \pi^{-1 / 2} \int_{0}^{x} y^{1 / 2} \exp (-y) d y$, where $x=v^{2} / v_{t h \beta}^{2}$ and $v_{t h \beta}$ is the thermal velocity of the background particles. The functions $F, G$ and $H$ are $F(x)=\left(1+m_{\alpha} / m_{\beta}\right) \Theta(x), G(x)=\Theta(x)[1-1 /(2 x)]+d \Theta / d x$ and $H(x)=\Theta(x) / x$. The test-particle drag and diffusion can be implemented by utilizing a Monte Carlo method due to $\mathrm{Xu}$ and Rosenbluth ${ }^{17}$. The particle weights are modified such that the collision operator annihilates a shifted Maxwellian ${ }^{16}$. Ion-electron collisions a Lorentz collision operator is used, and its Monte Carlo implementation has been discussed elsewhere ${ }^{19}$.

For the simulations presented in this paper, the trajectories of a set of $2 \times 10^{5}$ Lagrangian markers have been integrated ${ }^{20-22}$ with a time step $\Delta t / \tau_{i i}=4 \times 10^{-4}$. Collisional effects are calculated every 10 time steps. The confining $B$ field and the shape of the magnetic surfaces $(R, Z, \phi)$ have been specified in terms of Fourier series (Fig.3); a set of 30 Fourier harmonics have been retained in the calculations. Other parameters are the on-axis magnetic field $B_{0}=1.26 \times 10^{4}$ Gauss, central ion temperature $T_{i}(0)=2.76 \mathrm{KeV}$, central electron temperature $T_{e}(0)=2.14 \mathrm{KeV}$ and central plasma density $n_{0}=6.73 \times 10^{13} \mathrm{~cm}^{-3}$ (these parameters are the typical design parameters for NCSX). The magnetic surface of reference is located at $\bar{\psi} / \psi_{b}=0.7$. At each time step, the local (i.e. within $\delta \psi$; see Fig. 2) perpendicular and parallel pressures from each processor element (PE) are collected onto a single PE (say $\mathrm{PE}=0$ ); the Fourier coefficients for $P_{\|}$and $P_{\perp}$ are then calculated according to Eq.(7). All numerical parallel computations reported here have carried out with 16 PEs. 
The radial particle flux obtained from a direct measurement of the radial particle flux (arbitrary units) is shown as the jagged curve in Fig. 4 (in this case the applied radial electric field is 0 and the background distribution function $f_{0}$ has been loaded as a Maxwellian with $\left.\left\langle V_{\|}\right\rangle=0\right)$. It is noted that the noise is comparable to the signal; to determine the relation $\Gamma_{r}=\Gamma_{r}\left(E_{r}\right)$ from a direct measurement is not accurate. As a comparison, the thick line in Fig. 4 represents the radial particle flux calculated from the velocity moments $P_{\|}$and $P_{\perp}$.

The electron current density (calculated for large $t$ ) as a function of the normalized radial electric field $E_{r}=-\bar{a} d / d r\left(e \Phi / T_{i}(0)\right)$ is shown in Fig. 5. The electron current density displays an almost linear dependence on $E_{r}$. The ion current density, shown in Fig. 6, shows however a strong dependence on the $E_{r}$ parameter. The largest ion flux is obtained for $E_{r} \simeq-0.2$. We note that the ion flux is typically two orders of magnitude larger than the electron flux for small electric field (note the scale difference between Fig.5 and 6). By

inverting the relation $\Gamma_{i}\left[E_{r}^{(0)}\right]=\Gamma_{e}\left[E_{r}^{(0)}\right]$, we obtained $E_{r}^{(0)} \simeq-0.87$ statvolt $/ \mathrm{cm}$, that is $E_{r}^{(0)} \simeq-26.2 \mathrm{kV} / \mathrm{m}$ (which corresponds to the stable root). For illustrative purposes, the calculations have been carried out for a single magnetic surface (more precisely, for a single annulus), but a global calculation would lead the variation of $E_{r}$ with the radial coordinate.

\section{CONCLUDING REMARKS}

In this paper, we have presented a method to calculate the radial electric field in stellarator plasmas; our method is particularly useful for toroidal plasmas which depart weakly from axi-symmetry (e.g. the 'quasi-asymmetric concept'). It is has been shown that a direct measurement of the radial particle flux is very noisy, and not of much practical use for a direct calculation of $E_{r}$. The moment approach, however, shows a relatively smooth behavior and reaches an asymptotic value after a few ion-ion collision times. 
The method presented in this paper can be improved by including the off-diagonal terms (which are $\sim \rho_{i} / R_{0}$ times smaller than the diagonal terms) in the pressure tensor $\boldsymbol{\pi}$. However, since the dominant symmetry breaking term in the $B_{m n}$ spectrum is much larger than the smallness parameter $\rho_{i} / R_{0}$ one can, in first approximation, neglect the off-diagonal terms in the pressure tensor $\pi$.

An extension of the method presented in this paper can be used to determine the damping rates of toroidal and poloidal flows in stellarator plasmas; this is left for future work.

\section{ACKNOWLEDGMENTS}

One of us (J.L.V.L.) would like to thank W.W. Lee for encouragement. The method for carrying out reduced-noise $\delta f$ Monte Carlo calculations in single-helicity magnetic configurations is part of the doctoral thesis of one of us (J.W.). 


\section{APPENDIX A: DIVERGENCE OF THE PRESSURE TENSOR}

The pressure tensor, $\mathbf{P}$, can be written as

$$
\mathbf{P}=P_{\|} \widehat{\mathbf{b}} \widehat{\mathbf{b}}+P_{\perp}(\mathbf{I}-\widehat{\mathbf{b}} \widehat{\mathbf{b}})
$$

where $\widehat{\mathbf{b}}=\mathbf{B} / B$ is the unit vector along $\mathbf{B}, \mathbf{I}$ is the unit dyadic, and $P_{\|}$and $P_{\perp}$ are, respectively, the parallel and perpendicular pressures. We can also write Eq.(A1) as $\mathbf{P}=$ $\widetilde{P} \mathbf{B B}+P_{\perp} \mathbf{I}$, where $\widetilde{P} \equiv\left(P_{\|}-P_{\perp}\right) / B^{2}$. The divergence of the pressure tensor then reads

$$
\boldsymbol{\nabla} \cdot \mathbf{P}=\mathbf{B}(\mathbf{B} \cdot \boldsymbol{\nabla} \widetilde{P})+\widetilde{P} \boldsymbol{\nabla} \cdot(\mathbf{B B})+\boldsymbol{\nabla} P_{\perp}
$$

where the second term on the right-hand side can be calculated using the relation $\boldsymbol{\nabla} B^{2} / 2=$ $\mathbf{B} \times(\boldsymbol{\nabla} \times \mathbf{B})+(\mathbf{B} \cdot \boldsymbol{\nabla}) \mathbf{B}$ so that

$$
\nabla \cdot \mathbf{B B}=\frac{1}{2} \nabla B^{2}-\mathbf{B} \times(\nabla \times \mathbf{B})
$$

Using Ampere's law, $4 \pi \mathbf{J}=c \boldsymbol{\nabla} \times \mathbf{B}$, and the radial force balance, $\mathbf{J} \times \mathbf{B}=c \boldsymbol{\nabla} P_{0}$, one gets $\mathbf{B} \times(\boldsymbol{\nabla} \times \mathbf{B})=-4 \pi \boldsymbol{\nabla} P_{0}$ and the divergence of the pressure tensor now reads

$$
\boldsymbol{\nabla} \cdot \mathbf{P}=\mathbf{B}(\mathbf{B} \cdot \boldsymbol{\nabla} \widetilde{P})+\widetilde{P}\left(\frac{1}{2} \boldsymbol{\nabla} B^{2}+4 \pi \boldsymbol{\nabla} P_{0}\right)+\nabla P_{\perp}
$$

Taking the scalar product of Eq.(A4) with $\mathbf{e}_{\varphi} \equiv \partial \mathbf{r} / \partial \varphi$ where $\mathbf{r}$ is the position vector and $\varphi=\{\theta, \zeta\}$ one gets

$$
\mathbf{e}_{\varphi} \cdot(\boldsymbol{\nabla} \cdot \mathbf{P})=B_{\varphi}(\mathbf{B} \cdot \boldsymbol{\nabla} \widetilde{P})+\frac{\widetilde{P}}{2} \frac{\partial}{\partial \varphi} B^{2}+\frac{\partial P_{\perp}}{\partial \varphi}
$$

where we used the fact that the equilibrium pressure is a flux surface quantity, $P_{0}=P_{0}(\psi)$. In Boozer coordinates, the product of the Jacobian and the magnetic field strength squared is a flux surface quantity, that is $\mathcal{J} B^{2}=F(\psi)$, where $\mathcal{J}=[\boldsymbol{\nabla} \psi \cdot(\boldsymbol{\nabla} \theta \times \boldsymbol{\nabla} \zeta)]^{-1}$ denotes the Jacobian and $F(\psi)$ is a linear combination of the poloidal current, toroidal current and safety factor. We introduce the flux-surface average operator $\langle\bullet\rangle$ as

$$
\langle\bullet\rangle \equiv \iint \mathcal{J}(\bullet) d \theta d \zeta
$$


It is easy to show that $\langle\bullet\rangle$ annihilates the $\mathbf{B} \cdot \boldsymbol{\nabla}$ operator, that is

$$
\langle\mathbf{B} \cdot \nabla G\rangle=0
$$

for any function $G=G(\psi, \theta, \zeta)$. Noting that, in Boozer coordinates, $B_{\theta}$ and $B_{\zeta}$ are fluxsurface quantities, the flux-surface average of Eq.(A5) is

$$
\left\langle\mathbf{e}_{\varphi} \cdot(\boldsymbol{\nabla} \cdot \mathbf{P})\right\rangle=\frac{1}{2}\left\langle\frac{\partial}{\partial \varphi}\left(P_{\|}+P_{\perp}\right)\right\rangle,
$$

where we used Eq.(A7) and

$$
\begin{aligned}
\left\langle\widetilde{P} \frac{\partial B^{2}}{\partial \varphi}\right\rangle & =\left\langle\frac{P_{\|}-P_{\perp}}{B^{2}} \frac{\partial B^{2}}{\partial \varphi}\right\rangle \\
& =\left\langle B^{2}\left(P_{\perp}-P_{\|}\right) \frac{\partial}{\partial \varphi}\left(\frac{1}{B^{2}}\right)\right\rangle \\
& =\left\langle\frac{F(\psi)}{\mathcal{J}}\left(P_{\perp}-P_{\|}\right) \frac{\partial}{\partial \varphi}\left(\frac{1}{B^{2}}\right)\right\rangle \\
& =F(\psi) \iint\left(P_{\perp}-P_{\|}\right) \frac{\partial}{\partial \varphi}\left(\frac{1}{B^{2}}\right) d \theta d \zeta \\
& =F(\psi) \iint \frac{1}{B^{2}} \frac{\partial}{\partial \varphi}\left(P_{\|}-P_{\perp}\right) d \theta d \zeta \\
& =\iint \mathcal{J} \frac{\partial}{\partial \varphi}\left(P_{\|}-P_{\perp}\right) d \theta d \zeta \\
& =\left\langle\frac{\partial}{\partial \varphi}\left(P_{\|}-P_{\perp}\right)\right\rangle
\end{aligned}
$$




\section{REFERENCES}

${ }^{1}$ A. A. Galeev and R. Z. Sagdeev, in Reviews of Plasma Physics, edited by M.A. Leontovich (Consultants Bureau, New York, 1979), Vol. 7, p. 257.

${ }^{2}$ L.M. Kovrizhnykh, Nucl. Fusion 24, 851 (1984).

${ }^{3}$ A.H. Boozer, Phys. Fluids 24, 1999 (1981).

${ }^{4}$ A. Reiman, L. Ku, D. Monticello, et al, Plasma Physics and Controlled Nuclear Fusion Research, 1998 (International Atomic Energy Agency, Vienna, 1999), paper IAEA-CN69/ICP/06.

${ }^{5}$ G.H. Neilson, A.H. Reiman, M.C. Zarnstorff et al, Phys. Plasmas 7, 1911 (2000).

${ }^{6}$ G.Y. Fu, L.P. Ku, W.A. Cooper et al, Phys. Plasmas 7, 1809 (2000).

${ }^{7}$ M.H. Redi, A. Diallo, W.A. Cooper et al, Phys. Plasmas 7, 2508 (2000).

${ }^{8}$ K.H. Burrell, Phys. Plasmas 4, 1499 (1997).

9 T.S. Hahm and K.H. Burrell, Phys. Plasmas 2, 1648 (1995).

${ }^{10}$ A.H. Boozer, Phys. Plasmas 5, 1647 (1998).

11 A.M. Dimits and W.W. Lee, J. Comp. Phys. 107, 309 (1993).

12 S.E. Parker and W.W. Lee, Phys. Fluids. B5, 77 (1993).

${ }^{13}$ G. Hu and J.A. Krommes, Phys. Plasmas 1, 863 (1994).

${ }^{14}$ M. Sasinowski and A.H. Boozer, Phys. Plasmas 2, 610 (1995).

${ }^{15}$ Z. Lin, T.S. Hahm, W.W. Lee, W.M. Tang and R.B. White, Phys. Plasmas 7, 1857 (2000).

${ }^{16}$ Z. Lin, W. Tang and W.W. Lee, Phys. Plasmas 2, 2975 (1995).

${ }^{17}$ Q. Xu and M.N. Rosenbluth, Phys. Fluids B 3, 627 (1991).

18 M.N. Rosenbluth, P.H. Rutherford, J.B. Taylor, E.A. Frieman and L.M. Kovrizhnikh, 
in Plasma Physics and Controlled Nuclear Fusion Research 1971 (International Atomic Energy Agency, Vienna, 1971), Vol. I, p. 495.

${ }^{19}$ A. H. Boozer and G. Kuo-Petravic, Phys. Fluids 24, 851 (1981).

${ }^{20}$ R.B. White and M.S. Chance, Phys. Fluids 27, 2455 (1994).

${ }^{21}$ R.B. White, Phys. Fluids B2, 845 (1990).

${ }^{22}$ R.B. White and A.H. Boozer, Phys. Plasmas 2, 2915 (1995). 
Figure 1 Computational domain for $\delta f$ calculations of the radial electric field. The magnetic coordinates are $\psi, \theta$ and $\zeta$. The particles are initialized at the toroidal flux surface $\bar{\psi}$. The width of the annulus is such that $\delta \psi / \psi_{b} \ll 1$, where $\psi_{b}$ is the toroidal flux at the plasma boundary.

Figure 2 The Lagrangian $\delta f$ markers are uniformly distributed in the layer of width $\delta \Psi$; the radial width is chosen such that the typical drift time of an ion in the layer is larger than the relaxation time of the perturbed parallel and perpendicular calculated in the annulus $\delta \psi$ centered at $\bar{\psi}$.

Figure 3 Magnetic surface of the 3-field period National Compact Stellarator Experiment (NCSX). Although the shape of the magnetic surface is largely different for that of a comparable tokamak, the equilibrium $B$ field is approximately symmetric in $\zeta$ (magnetic toroidal angle) after transformation to Boozer coordinates.

Figure 4 Radial particle fluxes (arbitrary units) at the magnetic surface $\bar{\psi} / \psi_{b}=0.7$ as obtained from a direct measurement (thin, broken line) and from the fluid moment approach (thick line). The perpendicular and parallel pressures relax on a time scale of the order of a few ion-ion collision times. The applied radial electric field is zero and the time has been normalized to the ion-ion collision time $\tau_{i i}$.

Figure 5 Electron current density as a function of the normalized radial electric field $E_{r}=$ $-\bar{a} d / d r\left(e \Phi / T_{i}(0)\right)$, where $\bar{a}$ is the average minor radius of the last closed magnetic surface, and $T_{i}(0)$ is the ion temperature at the magnetic axis.

Figure 6 Ion current density as a function of the normalized radial electric field (plasma parameters are the same as in Fig.5). 
FIG.1 Lewandowski et al

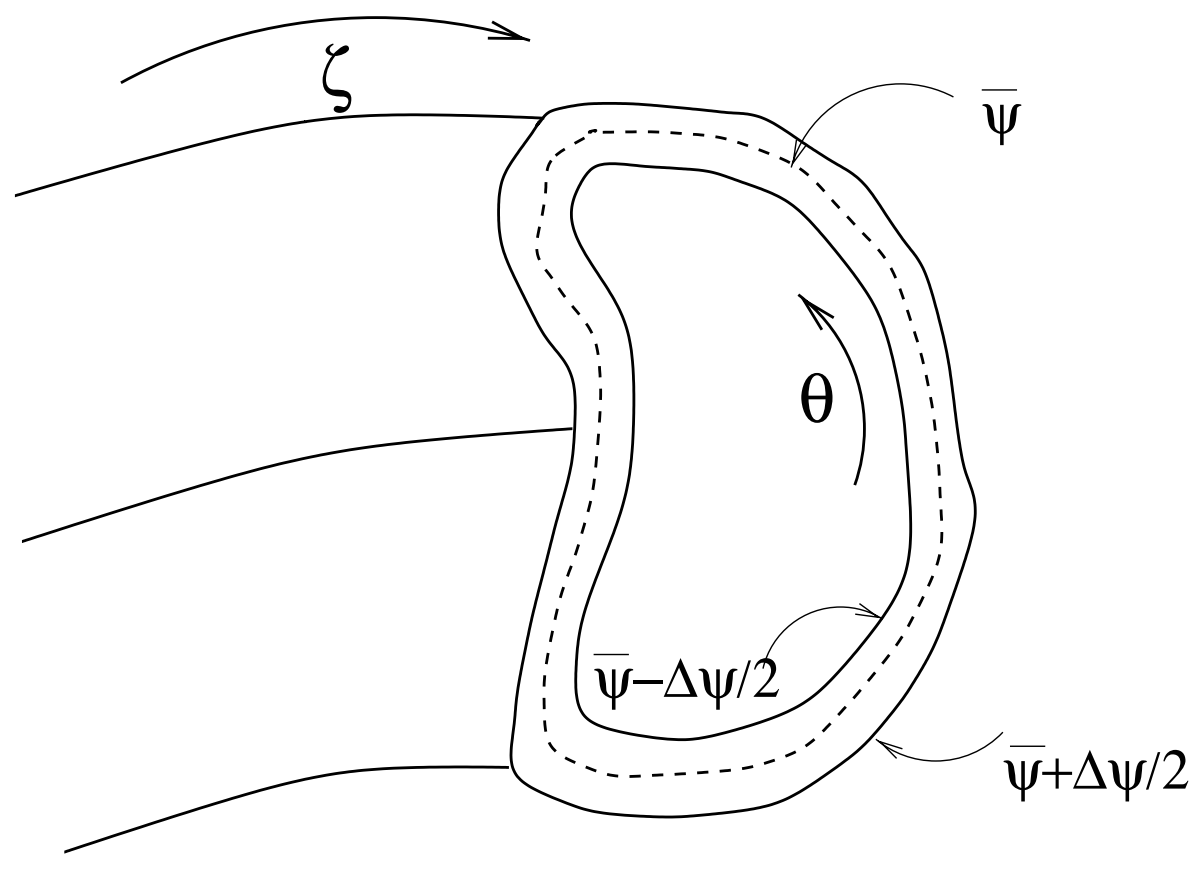


FIG.2 Lewandowski et al

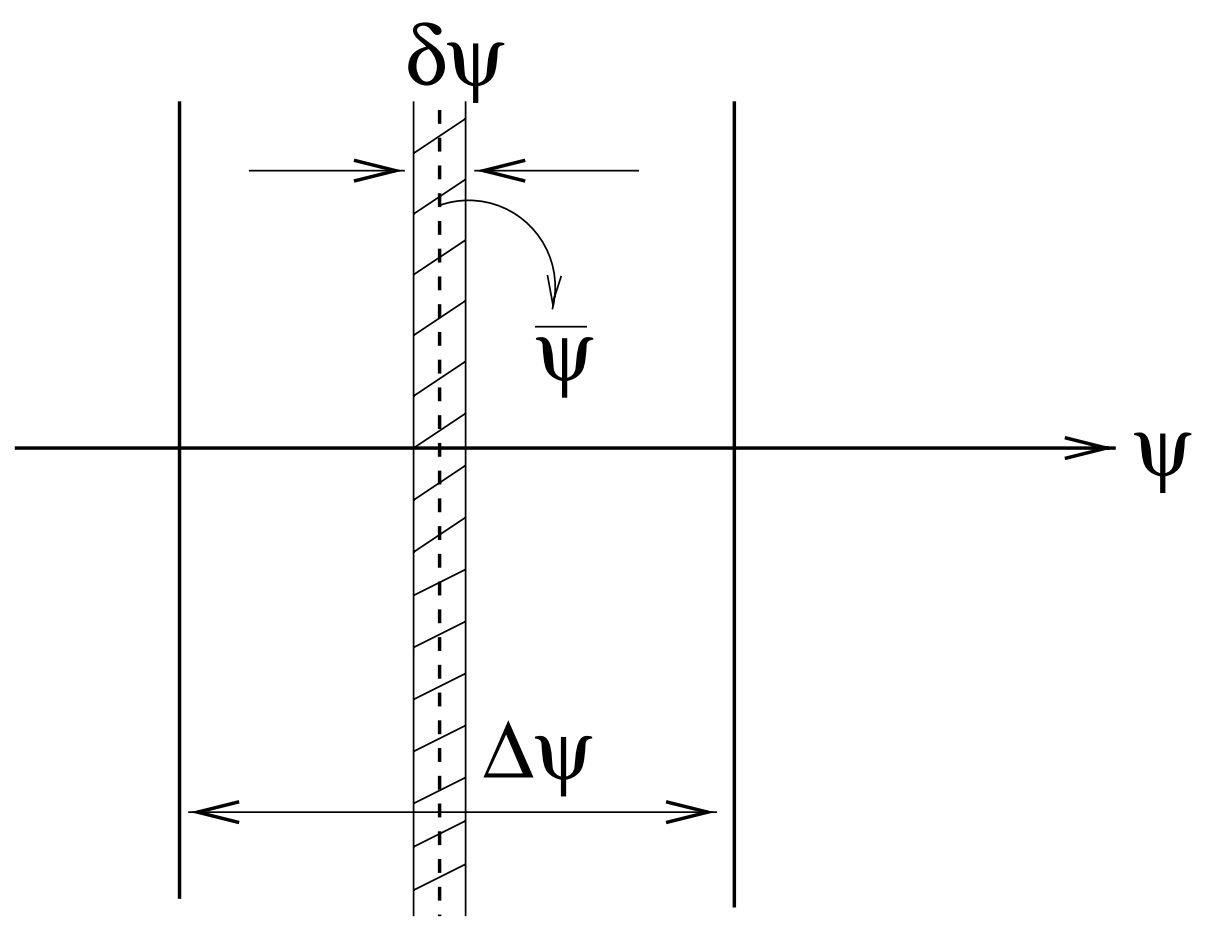


FIG.3 Lewandowski et al

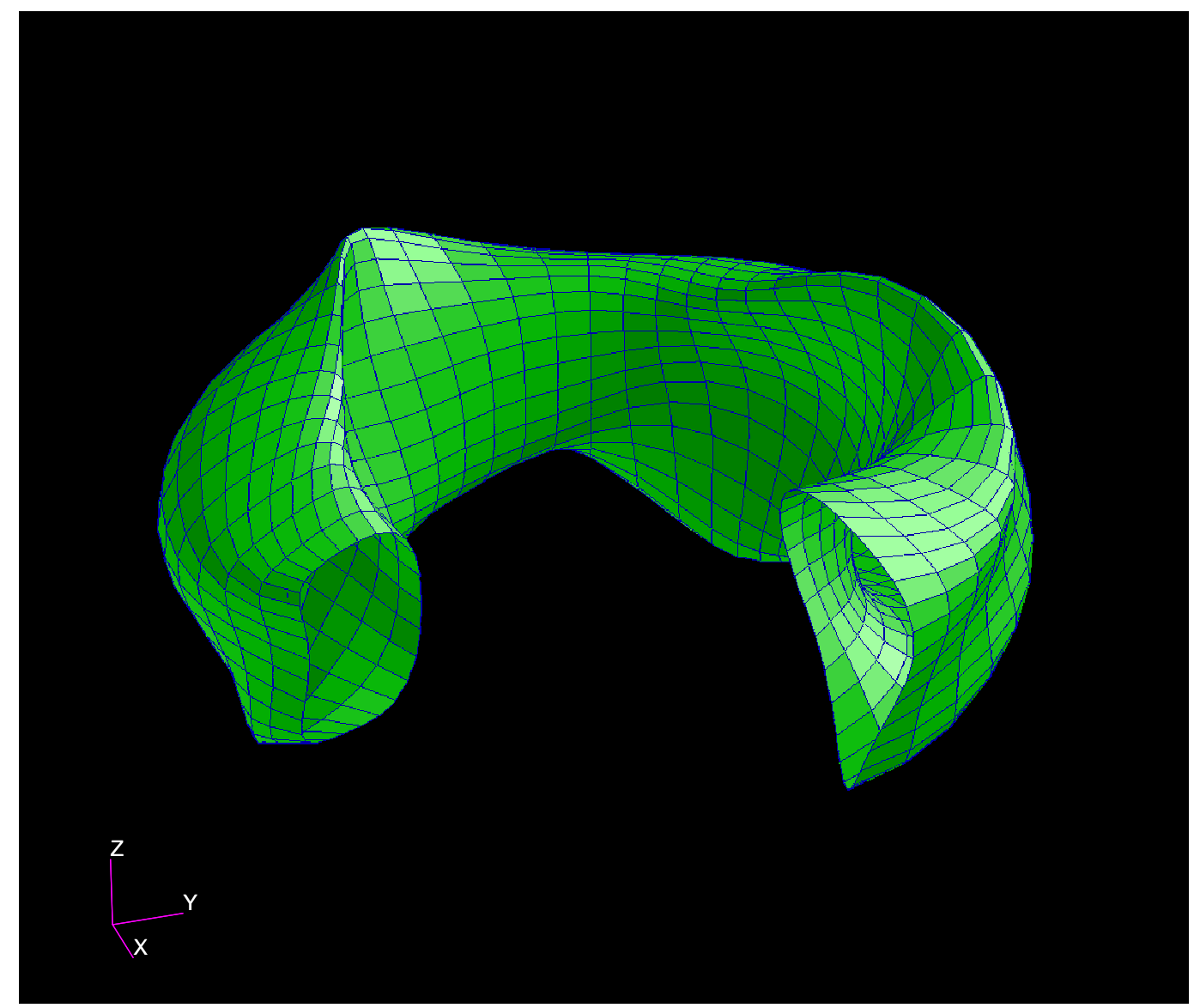


FIG.4 Lewandowski et al

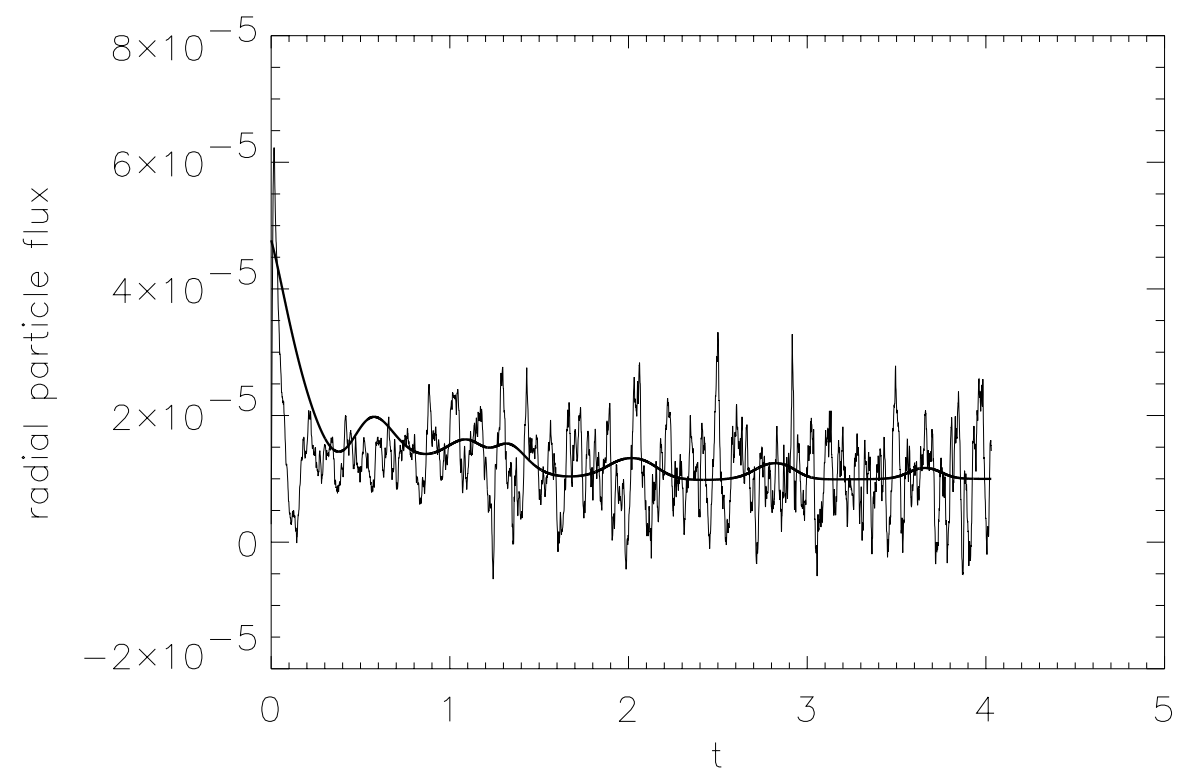


FIG.5 Lewandowski et al

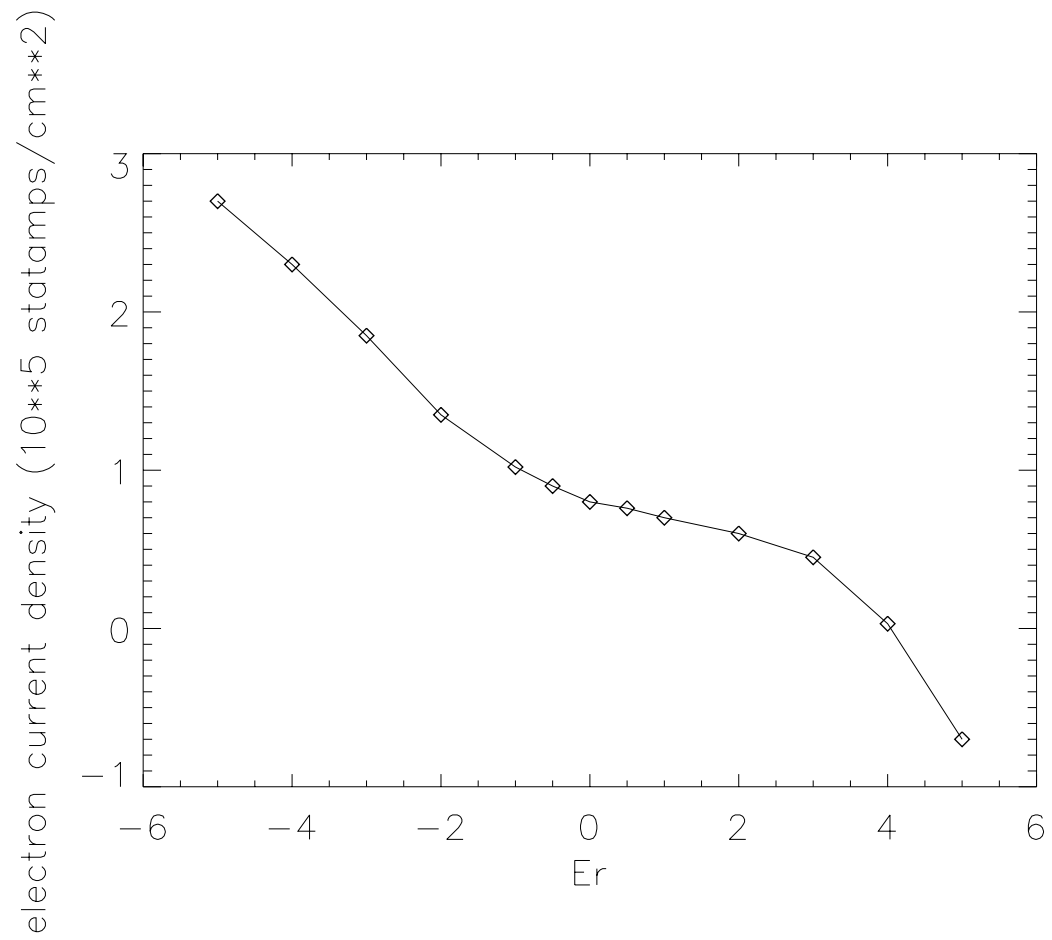


FIG.6 Lewandowski et al

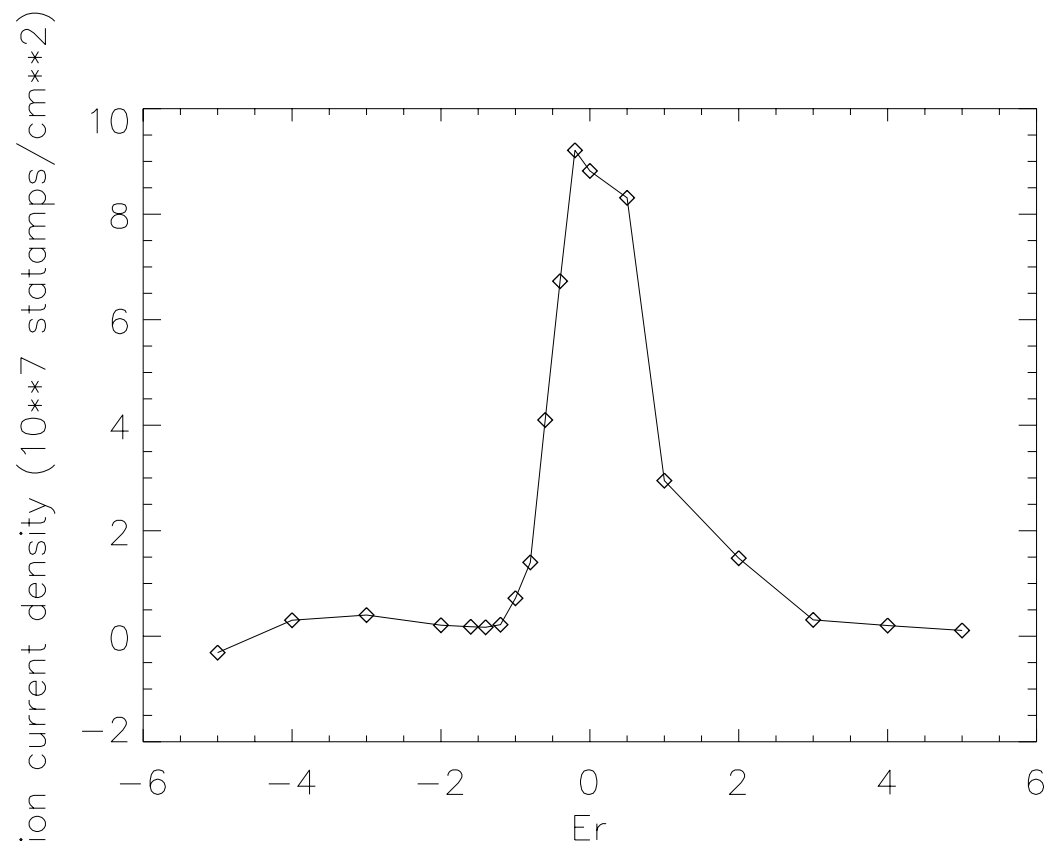




\section{External Distribution}

Plasma Research Laboratory, Australian National University, Australia

Professor I.R. J ones, Flinders University, Australia

Professor J oão Canalle, Instituto de Fisica DEQ/IF - UERJ , Brazil

Mr. Gerson O. Ludwig, Instituto Nacional de Pesquisas, Brazil

Dr. P.H. Sakanaka, Instituto Fisica, Brazil

The Librarian, Culham Laboratory, England

Library, R61, Rutherford Appleton Laboratory, England

Mrs. S.A. Hutchinson, JET Library, England

Professor M.N. Bussac, Ecole Polytechnique, France

Librarian, Max-Planck-Institut für Plasmaphysik, Germany

J olan Moldvai, Reports Library, MTA KFKI-ATKI, Hungary

Dr. P. Kaw, Institute for Plasma Research, India

Ms. P.J . Pathak, Librarian, Insitute for Plasma Research, India

Ms. Clelia De Palo, Associazione EURATOM-ENEA, I taly

Dr. G. Grosso, Instituto di Fisica del Plasma, Italy

Librarian, Naka Fusion Research Establishment, J AERI, J apan

Library, Plasma Physics Laboratory, Kyoto University, J apan

Research Information Center, National Institute for Fusion Science, J apan

Dr. O. Mitarai, Kyushu Tokai University, J apan

Library, Academia Sinica, Institute of Plasma Physics, People's Republic of China

Shih-Tung Tsai, Institute of Physics, Chinese Academy of Sciences, People's Republic of China

Dr. S. Mirnov, TRINITI, Troitsk, Russian Federation, Russia

Dr. V.S. Strelkov, Kurchatov Institute, Russian Federation, Russia

Professor Peter Lukac, Katedra Fyziky Plazmy MFF UK, Mlynska dolina F-2, Komenskeho Univerzita, SK-842 15 Bratislava, Slovakia

Dr. G.S. Lee, Korea Basic Science Institute, South Korea

Mr. Dennis Bruggink, Fusion Library, University of Wisconsin, USA

Institute for Plasma Research, University of Maryland, USA

Librarian, Fusion Energy Division, Oak Ridge National Laboratory, USA

Librarian, Institute of Fusion Studies, University of Texas, USA

Librarian, Magnetic Fusion Program, Lawrence Livermore National Laboratory, USA

Library, General Atomics, USA

Plasma Physics Group, Fusion Energy Research Program, University of California at San Diego, USA

Plasma Physics Library, Columbia University, USA

Alkesh Punjabi, Center for Fusion Research and Training, Hampton University, USA

Dr. W.M. Stacey, Fusion Research Center, Georgia Institute of Technology, USA

Dr. J ohn Willis, U.S. Department of Energy, Office of Fusion Energy Sciences, USA

Mr. Paul H. Wright, Indianapolis, Indiana, USA 
The Princeton Plasma Physics Laboratory is operated by Princeton University under contract with the U.S. Department of Energy.

\author{
Information Services \\ Princeton Plasma Physics Laboratory \\ P.O. Box 451 \\ Princeton, NJ 08543
}

Phone: 609-243-2750

Fax: 609-243-2751

e-mail: pppl_info@pppl.gov

Internet Address: http://www.pppl.gov 